\title{
Título de Especialista em Medicina Esportiva Sociedade Brasileira de Medicina do Esporte
}

\begin{abstract}
A Sociedade Brasileira de Medicina do Esporte (SBME) informa que estarão abertas no período de $\mathbf{0 2}$ de janeiro a $\mathbf{3 1}$ de março de 2007 (data de postagem) as inscrições para a prova para obtenção do Título de Especialista em Medicina Esportiva, emitido pela SBME e pela Associação Médica Brasileira (AMB).

O Título de Especialista em Medicina Esportiva (TEME) emitido pela SBME/AMB identifica o profissional médico com formação acadêmico-científica adequada e apto a exercer a especialidade com ética, responsabilidade e competência. Os critérios aqui estabelecidos estão de acordo com a Normatização da Concessão do Título de Especialista em Medicina Esportiva (4a revisão), publicada na Revista Brasileira de Medicina do Esporte em nov/dez de 2005, e estão de acordo com as exigências estabelecidas no convênio firmado entre o Conselho Federal de Medicina (CFM), a Associação Médica Brasileira (AMB) e a Comissão Nacional de Residência M édica em 11/04/2002; na Normativa de Regulamentação para Obtenção de Título de Especialista ou Certificado de Área de Atuação da AMB, de 13/01/2004; e na Resolução 1763/2005 do CFM. Conforme a Resolução 1772/2005 do CFM, o TEME - da mesma forma que todos os outros títulos de especialista - deverá passar pelo processo de atualização após 5 anos.

A prova para obtenção do TEME inclui a realização de uma Análise Curricular e uma Prova Escrita de caráter eliminatónio. A Prova Escrita será realizada no Hotel Intercontinental - Rio de J aneiro RJ , em sala a ser definida no local, no dia 02 de maio de 2007, das 09 às 12 horas; e das 14 às 17 horas.
\end{abstract}

\section{I - Documentos necessários para inscrição:}

1. Solicitação de inscrição assinada pelo candidato e encaminhada à Diretoria Científica da SBME constando nome completo, endereço residencial, endereço comercial, endereço de e-mail e telefones/fax para contato;

2. Cópia do documento de identidade do CRM;

3. Certidão de nada-consta emitida pelo CRM;

4. Curriculum vitae comprovado com os documentos adequados;

5. Comprovante dos itens 1 e 2 da parte II do presente edital;

6. Cheque nominal à SOCIEDADE BRASILEIRA DE MEDICI-

NA DO ESPORTE referente à taxa de inscrição no valor de $R$ \$ 400,00 (quatrocentos reais) ou de $R \$ 350,00$ (trezentos e cinqüenta reais) para os sócios quites da AMB, em situação regular e comprovada no ato da inscrição.

OBS 1 : A documentação deverá ser encaminhada em envelope claramente identificado "Prova de Titulo de Especialista em Medicina Esportiva" e assim endereçada:

Sociedade Brasileira de Medicina do Esporte

Diretoria Científica

Av. Brigadeiro Luiz Antonio, 278 - 40 andar

01318-901 (Centro) - São Paulo - SP

$\mathrm{OBS}_{2}$ : Os solicitantes que enviarem todos os documentos necessários receberão uma confimação de inscrição via fax, correio ou e-mail; entretanto, essa confirmação não tem caráter oficial, havendo a possibilidade por razões diversas do não recebimento por parte do candidato; assim sendo, o candidato deverá permanecer atento às datas e - caso não receba a confirmação - deverá entrar em contato com a Sociedade Brasileira de Medicina do Esporte, através do telefone/fax: (0xx11) 3106-7544.
OBS 3 : Serão considerados para a Análise Curricular somente os documentos enviados pelo correio e no ato da inscrição, não sendo aceita em hipótese alguma a adição posterior de documentos ou o envio de cópias de documentos por Fax, e-mail ou qualquer outro meio. A sua pontuação será computada previamente à prova escrita.

$\mathrm{OBS}_{4}$ : As remessas recebidas que não contiverem um ou mais dos itens acima não serão processadas e desta forma o solicitante não estará inscrito para a prova. Nestes casos, o cheque não será depositado, mas somente será devolvido ao próprio emitente em pessoa ou por correio, caso seja fornecido envelope previamente endereçado e selado. A devolução da documentação recebida estará submetida às mesmas condições. Caso a documentação não seja reclamada num prazo de 60 dias a mesma será destruída.

$\mathrm{OBS}_{5}$ : As inscrições que forem enviadas após 31 de março de 2007 (conforme a data do carimbo do correio) não serão processadas e desta forma o solicitante não estará inscrito para a prova; não serão aceitas solicitações de inscrição e cópias de documentos enviadas por Fax ou e-mail. Nessa situação a devolução da documentação segue as orientações da $\mathrm{OBS}_{4}$.

OBS $S_{6}$ Informações sobre a inscrição no evento que abrigará a prova (19o Congresso Brasileiro de Medicina do Esporte) podem ser obtidas através da empresa organizadora J Z Congressos, através do telefone (0x×21) 2266-9150 ou na própria Sociedade Brasileira de Medicina do Esporte, telefone/fax: (0xx11) 3106-7544.

$\mathrm{OBS}_{7}$ : A inscrição do candidato pressuporá o conhecimento e a incondicional aceitação das normas e condições estabelecidas neste Edital, em relação às quais não poderá alegar desconhecimento.

\section{II - Pré-requisitos para inscrição na Prova do TEME}

1. Ser formado há pelo menos 6 (seis) anos em Faculdade de Medicina reconhecida pelo órgão governamental competente e comprovar atuação efetiva na área de Medicina Esportiva durante esse período, caso não tenha concluído o Curso de Especialização em Medicina Esportiva ou ser formado há pelo menos 3 (três) anos em Faculdade de Medicina reconhecida pelo órgão governamental competente, caso já tenha concluído o Curso de Especialização em Medicina Esportiva, comprovando no mínimo 2 (dois) anos entre Especialização e atuação efetiva na área de M edicina Esportiva;

2. Estar regularmente inscrito, de forma definitiva, no CRM;

3. Não ter sido condenado em processo ético-profissional no CRM;

Obs.: os candidatos deverão satisfazer o pré-requisito disposto no item 1 no dia da realização da Prova Escrita.

\section{III - Critérios para aprovação na Prova do TEME}

1. Obter no mínimo 50\% de acerto na Prova Escrita; o candidato que não satisfizer este critério será eliminado, independentemente da pontuação total;

2. Obter no mínimo $60 \%$ do total de pontos possíveis, que corresponde ao somatório dos pontos da Análise Curricular e da Prova Escrita.

\section{IV - Provas}

O Exame compreenderá duas fases e obedecerá às seguintes disposições: 


\section{AVALIAÇÃO CURRICULAR}

Para a pontuação da Avaliação Curricular (máximo de 30 pontos), existe redundância, isto é, os pontos podem ser obtidos de diferentes formas, sendo até 15 pontos para formação profissional, até 10 pontos para experiência profissional e até 10 pontos para atividades acadêmicas, científicas e administrativas de acordo com a pontuação abaixo. TODOS OS ITENS DIZEM RESPEITO A ATIVIDADES OU TEMAS RELACIONADOS À MEDICINA DO EXERCÍCIO E DO ESPORTE.

\subsection{Formação profissional (até 15 pontos):}

Dentro da especialidade:

1.1.1. Estágios formais (> 100h)

1.1.2. Cursos de aperfeiçoamento organizados e/ou reconhecidos pela SBME, ou organizados pelas regionais reconhecidas pela SBME (carga horária igual ou superior a 30 horas)

1.1.3. Curso de Especialização reconhecido pelo Ministério da Educação, com carga horária igual ou superior a 360 horas

2 pontos

3 pontos

15 pontos

\subsection{Experiência profissional (até $\mathbf{1 0}$ pontos):}

a. Tempo de atuação profissional efetiva na especialidade:

1.2.1. 1 a 5 anos

3 pontos

1.2.2. 5 a 10 anos

1.2.3. mais de 10 anos

6 pontos

10 pontos

\subsection{Atividades acadêmicas, científicas e administrativas re-} lacionadas à especialidade (até 10 pontos):

a. Publicação de artigos como primeiro autor em periódicos indexados (por publicação) - máximo de 5 pontos:

1.3.1. no Brasil 1 ponto

1.3.2. na Revista Brasileira de Medicina do Esporte 3 pontos

1.3.3. no exterior

3 pontos

b. Publicação de capítulos de livros ou livros (por publicação) máximo de 5 pontos:

1.3.4. capítulos de livros no Brasil

1.3.5. editoria de livros no Brasil

1.3.6. capítulos de livros no exterior

1 ponto

2 pontos

1.3.7. editoria de livros no exterior

2 pontos

c. Apresentação e publicação de resumos - como primeiro autor - em eventos científicos (por evento) - máximo de 5 pontos:

1.3.8. no Brasil

1.3.9. em Congressos Brasileiros de Medicina

do Esporte (CBME)

1.3.10. no exterior

1 ponto

d. Participação em órgãos colegiados governamentais, câmaras técnicas profissionais ou conselhos editoriais de periódicos especializados e indexados (por ano) - máximo de 5 pontos:

1.3.11. local ou regional

1 ponto

1.3.12. nacional

2 pontos

1.3.13. internacional

3 pontos

e. Participação como ouvinte em congressos na especialidade, nos últimos 5 anos (por evento, máximo de 2 pontos):

1.3.14. congressos organizados pelas regionais reconhecidas pela SBME

1.3.15. Congressos Brasileiros de Medicina

do Esporte (CBME)

0,5 ponto

1.3.16. congressos no exterior

1 ponto

1 ponto

f. Participação docente em cursos e congressos (por evento):

1.3.17. aulas em cursos e jomadas

das regionais reconhecidas pela SBME 0,5 ponto (até 2 pontos)

1.3.18. participações no CBME como palestrante

1.3.19. participações no CBME como organizador

1 ponto (até 2 pontos)

2 pontos (até 4 pontos)
1.3.20. participações como palestrante

em congressos no exterior

g. Atividades administrativas (por gestão):

1.3.21. participação em diretoria de regional reconhecida pela SBME

2 pontos

\section{PROVA ESCRITA}

A Prova Escrita permitirá a totalização de 70 pontos, dos quais 45 pontos correspondem a questões de múltipla escolha e 25 pontos correspondem a questões discursivas e teórico-práticas.

A Prova Escrita terá caráter eliminatório. Os candidatos que não obtiverem ao menos $50 \%$ de acerto, ou seja, 35 pontos, serão sumariamente reprovados. Os candidatos que obtiverem pontuação igual ou superior a 50\% (35 pontos) terão essa pontuação somada à obtida na Avaliação Curricular. O gabarito da Prova Escrita será divulgado durante o 19 Congresso Brasileiro de Medicina do Esporte, evento que abriga a prova.

\section{PONTUAÇÃO GERAL E CRTÉRIO PARA APROVAÇÃO}

Os candidatos receberão até 30 pontos na Análise Curricular e até 70 pontos na Prova Escrita, perfazendo um total máximo possível de 100 pontos. Para aprovação, é necessário obter um mínimo de 35 pontos na Prova Escrita e ao mesmo tempo uma pontuação total de no mínimo 60 pontos.

\section{CONDIÇÕES PARA A REALIZAÇÃO DA PROVA ESCRITA}

4.1. A Prova Escrita será realizada no Hotel Intercontinental, na cidade do Rio de J aneiro, no dia 02 de maio de 2007, entre 9 e 12 horas; e entre 14 e 17 horas, em sala a ser definida no local.

4.2. O candidato deverá comparecer ao local da prova com pelo menos 01 (uma) hora de antecedência em relação ao horário previsto para o seu início.

4.3. Somente será admitido à sala da Prova Escrita o candidato que portar o original do seu documento oficial de identidade, sendo aceita Carteira expedida pelo Conselho Regional de Medicina do seu estado; Carteira expedida pela Secretaria de Segurança Pública do seu estado; Carteira expedida pelas Forças Armadas; Passaporte; Carteira Nacional de Habilitação emitida nos termos da Lei Federal no. 9503/97 (com foto); ou Carteira de Trabalho e Previdência Social. O documento apresentado deverá estar em perfeitas condições, de modo a permitir com clareza a identificação do candidato (retrato e assinatura). Não serão aceitos protocolos ou qualquer outro documento que impossibilite a clara identificação do candidato e a verificação de sua assinatura. Não haverá, sob qualquer pretexto, segunda chamada e nem será justificada falta, sendo considerado eliminado do exame o candidato que faltar à Prova Escrita.

4.4. Não será permitido, sob hipótese nenhuma, qualquer tipo de consulta a qualquer material que contenha informações sobre medicina, bem como porte e/ou utilização de meios eletrônicos que possibilitem comunicação à distância, e nem comunicação interpessoal.

4.5. Será eliminado da Prova o candidato que:

4.5.1. Ausentar-se da sala da Prova sem o acompanhamento do fiscal ou antes de decorrido o prazo fixado para saída;

4.5.2. Não devolver a Folha de Respostas e a Prova;

4.5.3. Durante a realização da Prova for surpreendido em comunicação com outro candidato ou com terceiros, através de qualquer forma;

4.5.4. For flagrado consultando livros, periódicos ou qualquer material ou meio eletrônico que contenha informações sobre medicina;

4.5.5. Estiver portando e/ou utilizando qualquer equipamento eletrônico de comunicação;

4.5.6. Lançar mão de qualquer meio ilícito para realizar a Prova. 
4.6. Não haverá prorrogação do tempo previsto para a realização da Prova em virtude de afastamento do candidato da sala da Prova, por qualquer motivo.

4.7. Os três últimos candidatos somente poderão sair juntos.

4.8. O candidato que não observar o disposto no item anterior, insistindo em sair do local da aplicação da Prova, deverá assinar termo desistindo da Prova e, caso se negue, deverá ser lavrado Termo de Ocorrência, testemunhado por dois outros candidatos, pelos fiscais e pelo executor.

4.9. O candidato deverá transcrever as respostas de todas as questões objetivas para a Folha de Respostas, que será o único documento válido para efeito de correção. O preenchimento da Folha de Respostas deverá ser realizado somente com caneta preta ou azul e será de inteira e exclusiva responsabilidade do candidato, que deverá proceder conforme as instruções contidas na capa do caderno de questões. Não haverá substituição da Folha de Respostas.

4.10. Na correção da Folha de Respostas será atribuída NOTA ZERO à questão:

a) com mais de uma opção assinalada;

b) sem opção assinalada;

c) com emenda ou rasura.

4.11. Os candidatos somente poderão se ausentar da sala da Prova após decorrida uma hora do seu início.

\section{V - Conteúdo programático da Prova Escrita}

1. Fisiologia do exercício: bioenergética, fisiologia muscular esquelética, efeitos agudos e crônicos sobre órgãos e sistemas, princípios da avaliação funcional e prescrição de exercícios;

2. Epidemiologia do exercício: histórico, papel da atividade física na prevenção primária de doenças, relação dose-resposta entre atividade física e redução da morbimortalidade;

3. Avaliação e condutas clínicas direcionadas a indivíduos que participam da prática de atividade física ou de exercícios competitivos: indivíduos aparentemente saudáveis, desportistas e atletas;

4. Avaliação e condutas clínicas direcionadas a indivíduos que participam da prática de atividade física e/ou de exercícios competitivos e fazem parte de grupos especiais: crianças, idosos, mulheres, portadores de doenças crônico-degenerativas (hipertensos, miocardiopatas, coronariopatas, diabéticos, pneumopatas crônicos, nefropatas, portadores de doenças neurológicas, etc.) e portadores de deficiências;

5. Ortopedia e traumatologia desportiva com ênfase nas medidas de prevenção e reabilitação de lesões;

6. Cineantropometria;

7. Doping e controle anti-doping;

8. Cardiologia do esporte.

\section{VI - Bibliografia sugerida}

- Amatuzzi M M, Carazzato J G. (eds). Medicina do Esporte. São Paulo: Rocca, 2004.

- Carvalho T, Nóbrega ACL, Lazzoli J K, Magni J RT, Rezende L, Drummond FA et al. Posicionamento oficial da Sociedade Brasileira de Medicina do Esporte sobre atividade física e saúde. Rev Bras Med Esporte 1996; 2: 79-81.

- $\mathrm{Fu}, \mathrm{FH}$, Stone DA. (eds.). Sports injuries: mechanisms, prevention $\&$ treatment. $2^{\text {nd }}$ edition. Philadelphia: Lippincott Williams $\&$ Wilkins, 2001.

- Froelicher VF, Myers J N. Exercise and the heart. $4^{\text {th }}$ edition, Philadelphia: WB Saunders, 2000.

- Ghorayeb N, Barros Neto TL (eds.). O exercício: preparação fisiológica, avaliação médica, aspectos especiais e preventivos. São Paulo: Editora Atheneu, 1999.

- Ghorayeb N, Dioguardi GS. Tratado de cardiologia do exercício e do esporte. São Paulo: Editora Atheneu, 2006.
- Lazzoli J K, Nóbrega AC, Carvalho T, Oliveira MAB, Teixeira J AC, Leitão MB et al. Posicionamento oficial da Sociedade Brasileira de Medicina do Esporte sobre atividade física e saúde na infância e adolescência. Rev Bras Med Esporte 1998; 4: 107-9.

- Leitão MB, Lazzoli J K, Oliveira MAB, Nóbrega ACL, Silveira GG, Carvalho T et al. Posicionamento Oficial da Sociedade Brasileira de Medicina do Esporte sobre atividade física e saúde na muIher. Rev Bras Med Esporte 2000; 6: 215-20.

- Maron BJ , Zipes DP. 36th Bethesda Conference: eligibility recommendations for competitive athletes with cardiovascular abnormalities. J Am Coll Cardiol 2005; 45: 1313-75.

- Nóbrega ACL, Freitas EV, Oliveira MAB, Leitão MB, Lazzoli J K, Nahas RM et al. Posicionamento Oficial da Sociedade Brasileira de Medicina do Esporte e da Sociedade Brasileira de Geriatria e Gerontologia sobre atividade física e saúde no idoso. Rev Bras Med Esporte 1999; 5: 207-11.

- Oliveira MAB, Nóbrega ACL, eds. Clínicas Brasileiras de Medicina do Esporte - Tópicos Especiais em Medicina do Esporte: v. 1. São Paulo: Atheneu, 2003.

- Pancorbo Sandoval, AE. Medicina do Esporte - princípios e prática. Porto Alegre: Artmed, 2005.

- Pelliccia A, Fagard R, Bjørnstad HH, Anastassakis A, Arbustini $H$, Assanelli $D$ et al. Recommendations for competitive sports participation in athletes with cardiovascular disease - a consensus document from the Study Group of Sports Cardiology of the Working Group of Cardiac Rehabilitation and Exercise Physiology and the Working Group of Myocardial and Pericardial Diseases of the European Society of Cardiology. Eur HeartJ 2005; 26: 1422-45.

- Pollock ML, Schmidt DH. Doença cardíaca e reabilitação. 3a edição. Rio de J aneiro: Revinter, 2003.

- Sociedade Brasileira de Medicina do Esporte. I Consenso de Petrópolis: Posicionamento Oficial sobre esporte competitivo em indivíduos acima de 35 anos. Rev Bras Med Esporte 2001; 7: 8392.

- Sociedade Brasileira de Medicina do Esporte. Diretriz sobre modificações dietéticas, reposição hídrica, suplementos alimentares e drogas: comprovação de ação ergogênica e potenciais riscos para a saúde. Rev Bras Med Esporte 2003; 9: 43-56.

- Sociedade Brasileira de Medicina do Esporte. Diretriz sobre morte súbita no exercício e no esporte. Rev Bras Med Esporte 2005; 11(supl 1): S1-8.

- Whaley MH, Brubaker PH, Otto RM (eds.). ACSM's Guidelines for exercise testing and prescription., $7^{\text {th }}$ edition, Philadelphia: Lippincott Williams \& Wilkins, 2006.

\section{VI - Recursos de Candidatos Reprovados}

Os candidatos reprovados que desejarem, poderão submeter, por escrito, um recurso à Comissão, que decidirá, em caráter irrevogável dentro do âmbito da SBME, sobre a manutenção ou não do resultado. O prazo para apresentação do recurso é de 72 horas após a divulgação dos resultados.

\section{VII - Emissão do Título de Especialista em Medicina Espor- tiva}

Os candidatos aprovados receberão uma declaração emitida pela SBME constando a data de aprovação. A emissão do TEME estará condicionada ao recebimento da taxa de confecção, conforme critérios da própria AMB.

São Paulo, 15 de dezembro de 2006.

Dr. J osé Kawazoe Lazzoli Diretor Científico

Presidente da Comissão do TEME Sociedade Brasileira de Medicina do Esporte 